ADLFI. Archéologie de la France -

INFORMATIONS Informations

une revue Gallia

Rhône-Alpes | 2009

\title{
La Rivière
}

Ancienne bergerie de l'alpage de Fessole - domaine des Ecouges

Alain Belmont

\section{(2) OpenEdition}

Édition électronique

URL : http://journals.openedition.org/adlfi/3444

ISSN : 2114-0502

Éditeur

Ministère de la culture

Référence électronique

Alain Belmont, «La Rivière », ADLFI. Archéologie de la France - Informations [En ligne], Rhône-Alpes, mis en ligne le 01 mars 2009, consulté le 03 mai 2019. URL : http://journals.openedition.org/adlfi/3444

Ce document a été généré automatiquement le 3 mai 2019.

(c) Ministère de la Culture et de la Communication, CNRS 


\title{
La Rivière
}

\author{
Ancienne bergerie de l'alpage de Fessole - domaine des Ecouges
}

\section{Alain Belmont}

Identifiant de l'opération archéologique : 9974

Date de l'opération : 2009 (FP)

1 Le LARHRA (UMR 5190 CNRS-Université Grenoble 2) effectue depuis 2005 des recherches systématiques sur l'occupation et les activités humaines entre le Moyen Âge et le XIX $\mathrm{X}^{\mathrm{e}}$., d'un ancien « désert » cartusien implanté en partie nord du plateau du Vercors, dans la vallée des Ecouges. Au cours de ce programme, quatre sites ont ainsi été fouillés ou sondés : une carrière de meules des XIII ${ }^{\mathrm{e}}$ s.-XV $\mathrm{XV}^{\mathrm{e}}$ s., un moulin à eau des XII ${ }^{\mathrm{e}}$ s.-XV ${ }^{\mathrm{e}}$ s., une ferme des $\mathrm{XVII}^{\mathrm{e}} \mathrm{s}$.-XX ${ }^{\mathrm{e}}$ s., une charbonnière et un entrepôt à charbons de bois des XIIV ${ }^{\mathrm{e}}$ s.$\mathrm{XIX}^{\mathrm{e}} \mathrm{s}$. Une prospection pédestre menée sur 500 ha a également permis la découverte de plus de 250 sites archéologiques, avec dans le lot l'ancien monastère des Chartreux (XII $\left.\mathrm{s} .-\mathrm{XV}^{\mathrm{e}} \mathrm{s}.\right)$, le troisième fondé par l'ordre de saint Bruno, dont une prospection géophysique révéla l'ampleur tout à fait inattendue.

2 La campagne 2009 s'est attachée à une grande structure de pierres découverte deux ans plus tôt en prospection prédestre, dans l'alpage couronnant le sommet de la vallée, à $1400 \mathrm{~m}$ d'altitude. Trois sondages d'une surface totale de $79 \mathrm{~m}^{2}$ ont été ouverts, deux à plan en $\mathrm{L}$ à l'intérieur du bâtiment, et le troisième à l'extérieur et le long du mur gouttereau sud. Ils ont révélé un édifice à plan rectangulaire de $19 \mathrm{~m}$ de long pour $13,5 \mathrm{~m}$ de large, appuyé à l'est sur une petite falaise et cerné par de puissants murs de $1 \mathrm{~m}$ d'épaisseur (Fig. $\mathrm{n}^{\circ} 1$ : plan des bâtiments XIIe -XVIIIe s.). Ceux-ci, construits en moellons de petit et moyen appareil liés par un mortier de terre et de chaux, ont été édifiés en deux étapes et s'élevaient au moins à 3,60 m de haut. Le tout était probablement surmonté d'un étage faisant office de fenil, couvert par un toit d'essendoles (des tuiles de bois). L'accès se faisait par une porte ouverte en pignon ouest et peut-être aussi par un "montoir » aménagé en pignon oriental. L'intérieur du bâtiment offrait une surface disponible de $195 \mathrm{~m}^{2}$. Cet espace, d'abord d'un seul tenant, présentait un sol très compact fait 
d'argile et de gravier de silex, et dont les pentes convergeaient vers une fosse de $5 \mathrm{~m}$ de diamètre creusée au centre du bâtiment. Le sol d'occupation était recouvert d'un niveau d'incendie et a fourni très peu de mobilier archéologique. Lors de la deuxième ou d'une troisième campagne de construction, une pièce d'une surface de $50 \mathrm{~m}^{2}$ fut aménagée à l'intérieur du bâtiment et de telle sorte qu'elle s'appuie contre la falaise et soit entièrement isolée des murs d'enceinte extérieurs (Fig. $n^{\circ} 2$ : vue générale du site).

À la lumière du plan obtenu et des informations glanées dans les textes d'archives, le site de Fessole est identifié comme une ancienne bergerie, à l'intérieur de laquelle fut aménagée ultérieurement une fromagerie. La première phase de construction, datée par ${ }^{14} \mathrm{C}$, est contemporaine de l'installation des chartreux aux Ecouges au début du XII ${ }^{\mathrm{e}} \mathrm{s}$; ; ce premier bâtiment fut incendié quelques décennies plus tard, peut-être à l'occasion de graves litiges ayant opposé les chartreux aux seigneurs des environs au cours des années 1190. Un siècle et demi après que les chartreux aient abandonné leur monastère des Ecouges, la bergerie de Fessole est reconstruite entre 1657 et 1665 par Laurence Frère, veuve d'un président au parlement de Dauphiné et dont la famille a obtenu l'albergement de la vallée par l'évêché de Grenoble. Ce changement de propriétaire s'accompagne d'une mutation des pratiques pastorales, puisque l'élevage ovin de l'époque médiévale cède la place à un troupeau d'une centaine de vaches. Leur lait est transformé en fromages par des spécialistes venus de Suisse, d'où l'aménagement de la fromagerie à l'intérieur du bâtiment. Le " habert » de Fessole fait les frais d'une rivalité entre les propriétaires du lieu et la commune de La Rivière, désireuse d'accéder aux alpages ; il est détruit lors de la Révolution française, aux alentours de 1793-94.

La campagne de 2009 a enfin mis au jour un trou de poteau daté par ${ }^{14} \mathrm{C}$ des VIII $\mathrm{s}$.- $\mathrm{X}^{\mathrm{e}}$ $\mathrm{s}$. $\mathrm{AD}$, et qui a pu appartenir à un bâtiment de terre et de bois antérieur à la construction de la bergerie par les chartreux. Ce sont donc au moins mille ans d'occupation pastorale qui se sont déroulés sur ce site de moyenne montagne. Il témoigne des évolutions intervenues sur le long terme, avec le passage d'un élevage ovin à un élevage bovin, avec l'introduction des fromagers suisses révélatrice d'un perfectionnement des techniques et d'une ouverture des débouchés commerciaux vers de larges horizons, et montre enfin l'importance des investissements que les chartreux à l'époque médiévale et la grande noblesse provinciale à l'époque moderne, étaient prêts à réaliser pour ces vastes équipements, à la pointe du progrès de leur temps. 
Fig. $n^{\circ} 1$ : plan des bâtiments XIIe -XVIIle s.

\section{Les Ecouges (38) \\ Ferme de Fessole}

Plan général

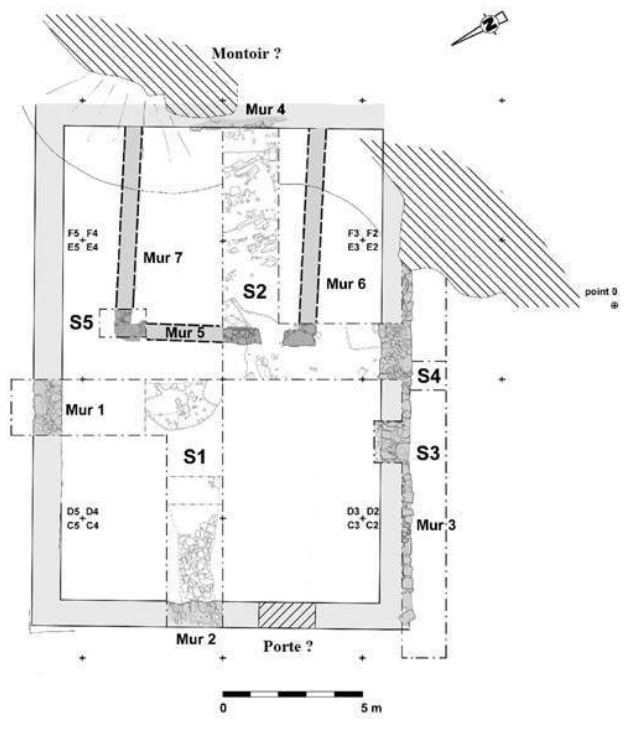

Auteur(s) : Penon, S. (LARHRA) ; Belmont, Alain (Université de Grenoble LARHRA). Crédits : S. Penon LARHRA (2009)

\section{Fig. $n^{\circ} 2$ : vue générale du site}

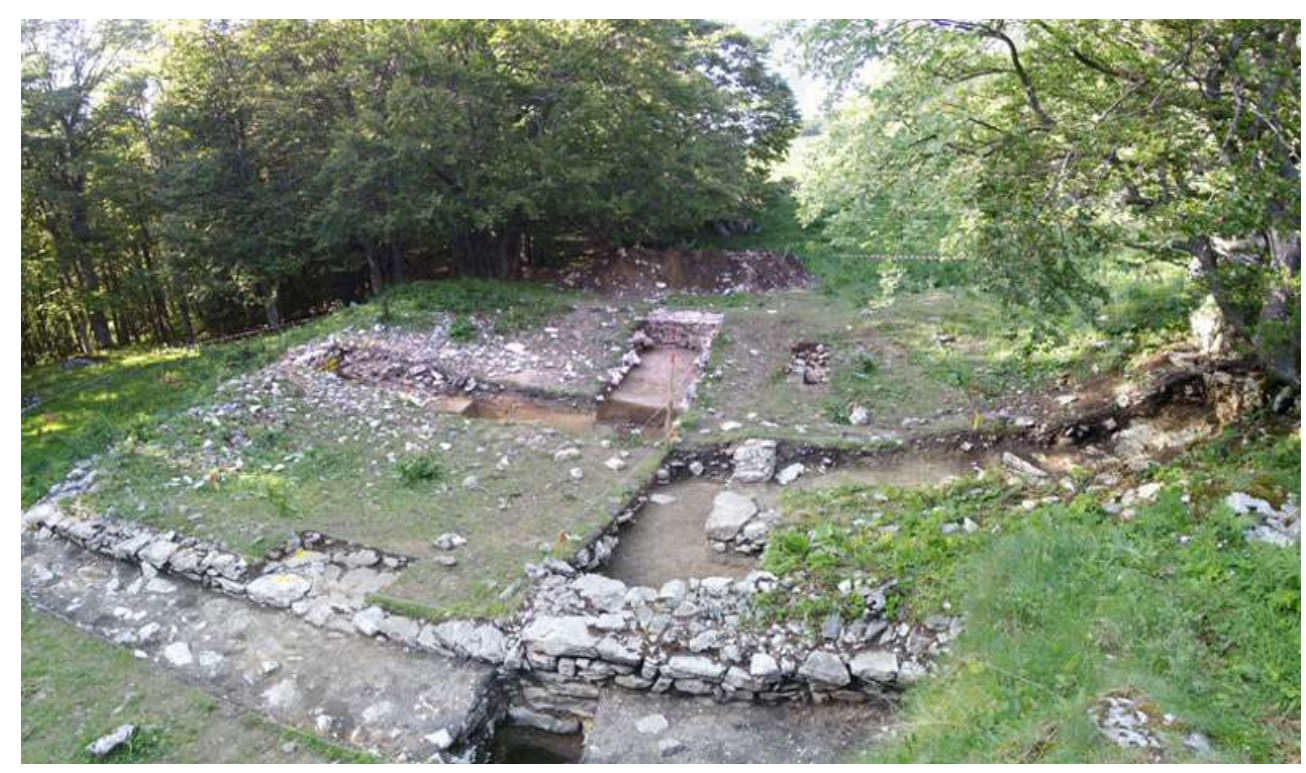

Auteur(s) : Minvielle, Nicolas (LARHRA). Crédits : N. Minvielle LARHRA (2009) 
INDEX

Thèmes : bâtiment agricole, bergerie, Chartreux, fromage, monastère

Index chronologique : IXe siècle apr. J.-C., Moyen Âge, VIIIe siècle apr. J.-C., Xe siècle apr. J.-C., XIe siècle apr. J.-C., XIIe siècle apr. J.-C., XVIIIe siècle apr. J.-C.

Index géographique : Rhône-Alpes, Isère, Rivière

operation fouille programmée (FP)

\section{AUTEUR}

ALAIN BELMONT 